\title{
In-Vitro Biofilm Detection among Uropathogens and Their Antibiogram Profile
}

\author{
Bijayata Shrestha ${ }^{1 *}$, Basudha Shrestha ${ }^{2}$, Asia Poudel ${ }^{1}$ Binod Lekhak $^{3}$, Milan Kumar Upreti ${ }^{1}$ \\ Department of Microbiology ${ }^{1}$, GoldenGate international College, Kathmandu, Nepal \\ Department of Pathology², Kathmandu Model Hospital, Baghbazaar, Kathmandu, Nepal \\ Central Department ${ }^{3}$ of Microbiology, Kirtipur, Nepal
}

\begin{abstract}
*Corresponding author: Bijayata Shrestha, Department of Microbiology, GoldenGate International College, E-mail: bijayatastha1@gmail.com
\end{abstract}

\begin{abstract}
Objectives: The study was carried out in Kathmandu Model Hospital, Kathmandu with the aim of in-vitro biofilm detection among uropathogens and its correlation with antibiotic resistance.

Methods: Uropathogens $(n=234)$ were isolated, and identified with standard microbiological techniques and further subjected to Modified Congo Red Agar Method for the biofilm detection in-vitro; antimicrobial susceptibility testing (10 antibiotics) was performed by Modified Kirby Bauer disc diffusion method. The MIC and MBEC values of Levofloxacin were determined by agar dilution for planktonic forms and by microdilution method for biofilm phase respectively.

Results: Among 234 urine isolates, 134(57\%) were positive for in-vitro biofilm production and $88(37.6 \%)$ were multidrug resistant (MDR). E.coli was the predominant biofilm forming uropathogens. The incidence of biofilm producers was found to be independent of age-wise, gender wise and indoor-outdoor distribution of patients. The association between biofilm production and multidrug resistance among uropathogens was found statistically non-significant ( $p$-value>0.05). The MBEC values of biofilm phase of growth were found to be greater than the MIC values for their planktonic counterparts. The MBEC values ranged from 4 to more than $1024 \mu \mathrm{g} / \mathrm{ml}$ whereas the MIC values ranged from $0.003-16 \mu \mathrm{g} / \mathrm{ml}$.

Conclusion: The results of this study suggest that biofilm detection is a critical step to fight against biofilm-involved infections. However, further studies are needed for the development of effective preventive and treatment strategies of biofilm associated UTIs to avoid recurrence and persistence.
\end{abstract}

Key words: Biofilms, UTI, MIC, MBEC, Multidrug Resistance.

\section{INTRODUCTION}

As human prefers to live in community, microorganisms that affect human life in so many ways also prefer to exist in a community. Such microbial community attached to a surface that may be biotic or abiotic, embedded in a self-produced extracellular polymeric matrix is referred to as "Biofilm" (Donlan and Costerton 2002). As per NIH (2002), about $65-80 \%$ of human infections are caused by biofilm forming organisms.

UTI is the most common infection encountered in the community as well as hospital settings and is

Date of Submission: November 12, 2018

Published Online: January 2019 often associated with the problems of recurrence and persistence (Soto et al. 2007; Ejrnaes et al. 2011). Chronic and recurrent infections are usually caused by biofilm associated pathogens which are recalcitrant to standard antibiotic therapy (Hancock et al. 2007; Ejrnaes et al. 2011). Bacteria in biofilms are phenotypically different from their planktonic counterparts and exhibit higher antibiotic resistance, leading to treatment failure and recovery from the infection very difficult (Choong and Whitefield, 2000). Thus, treatment of biofilm associated infections should target biofilms rather than their planktonic counterparts (Kostakioti et al. 2013).

Date of Acceptance: December 10, 2018

DOI: https:/ / doi.org/10.3126/tujm.v5i0.22313 
The main objective of the study was to determine the proportion of biofilm producers among uropathogens and their antibiogram profile, the knowledge of which can guide towards effective management of biofilm associated UTI and help in prevention of recurrent and persistent UTI.

\section{MATERIALS AND METHODS}

\section{Study site and population}

This study was carried out in Department of Pathology of Kathmandu Model Hospital in collaboration with Department of Microbiology of GoldenGate International College. Ethical approval for the study was taken from Institutional Review Committee phect Nepal. A total of 1299 midstream urine from UTI suspected patients received for culture were included in the study.

\section{Urine culture}

Semi-quantitative urineculture was performed on CLED agar using a standardized calibrated loop $(0.001 \mathrm{ml})$. The agar plate was incubated at $37^{\circ} \mathrm{C}$ for 24 hours and then observed for colonial count. Colonial count greater than $10^{5} \mathrm{cfu} / \mathrm{ml}$, was considered significant.

\section{Isolation and identification of bacterial isolates}

The isolates were identified based on the standard microbiological procedures which included colonial appearance, staining reaction and biochemical properties.

\section{Antibiotic susceptibility testing}

The antibiotic susceptibility testing was done by modified Kirby Bauer disc diffusion technique. Antibiotics were selected as per CLSI (2013) guidelines.

\section{Screening of biofilm producers}

The isolate was streaked over modified Congo Red agar and incubated at $37^{\circ} \mathrm{C}$ for 24 hours and observed for black coloured colonies.

\section{Determination of MICs and MBECs}

MICs of Levofloxacin for all the isolates were determined by agar dilution method (EUCAST, 2000). MBECs of Levofloxacin for the sessile form of biofilm forming isolates were determined using microdilution method, a modification of method described by Ghanwante (2012).

\section{Quality control}

E.coli ATCC 25922, Staphylococcus aureus ATCC 25923 and Pseudomonas aeruginosa ATCC 27853 were used as control strains.

\section{Data analysis}

Data were analyzed using SPSS version 16. p-value less than 0.05 was considered significant.

\section{RESULTS}

Out of 1299 urine specimens, 234(18\%) showed significant growth. The most commonly isolated organism from UTI patients was E. coli, accounting for $78.6 \%$ of the total isolates.

Table 1: Bacterial isolates among UTI patients

\begin{tabular}{lc}
\hline Bacterial isolates & Total $(\mathrm{n}=234)$ \\
\hline E. coli & $184(78.6 \%)$ \\
Klebsiella spp. & $8(3.4 \%)$ \\
Proteus spp. & $2(0.8 \%)$ \\
P. aeruginosa & $2(0.8 \%)$ \\
A. baumannii & $2(0.8 \%)$ \\
Staphylococcus spp. & $23(9.8 \%)$ \\
Enterococcus faecalis & $8(3.4 \%)$ \\
Enterobacter spp. & $2(0.8 \%)$ \\
Citrobacter spp. & $2(0.8 \%)$ \\
Salmonella Typhi & $1(0.4 \%)$ \\
\hline
\end{tabular}

Out of 234 uropathogens, 57\% (134) were biofilm producers among which E.coli $(90 \%)$ constituted the highest percentage. Other organisms involved in biofilm production were Klebsiella spp., Acinetobacter baumannii, Staphylococcus spp. and Enterococcus faecalis. 


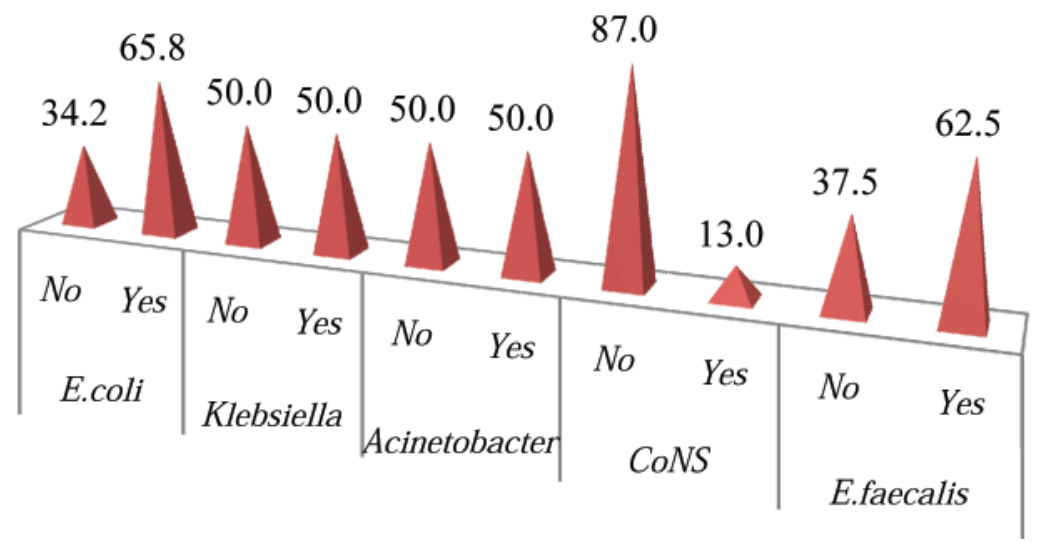

Figure 1: Biofilm producers among different uropathogens

The incidence of biofilm producers was similar among male and female patients. The percentage of biofilm producers was slightly higher among in-patients (62.5\%) as compared to out-patients (56.7\%). However, biofilm production among uropathogens was statistically nonsignificant to sex-wise and indoor- outdoor distribution of patients ( $p$-value $>0.05$ ).

Table 2: Pattern of biofilm forming uropathogens among patients

\begin{tabular}{|c|c|c|c|c|c|}
\hline \multirow{2}{*}{ Biofilm } & \multicolumn{2}{|c|}{$\operatorname{Sex}(p>0.05)$} & \multicolumn{2}{|c|}{ Patient type $(p>0.05)$} & \multirow{2}{*}{ Total $(n=234)$} \\
\hline & Male $(n=66)$ & Female $(n=168)$ & Outpatient $(n=210)$ & Inpatient (n=24) & \\
\hline Producer & $38(57.7 \%)$ & $96(57.1 \%)$ & $119(56.7 \%)$ & $15(56.7 \%)$ & 134 \\
\hline Non-producer & $38(43.3 \%)$ & $72(42.9 \%)$ & $91(43.3 \%)$ & $9(37.5 \%)$ & 100 \\
\hline
\end{tabular}

Though biofilm producers were present among patients of all age groups, the number was highest among the

age group 20-30 years.

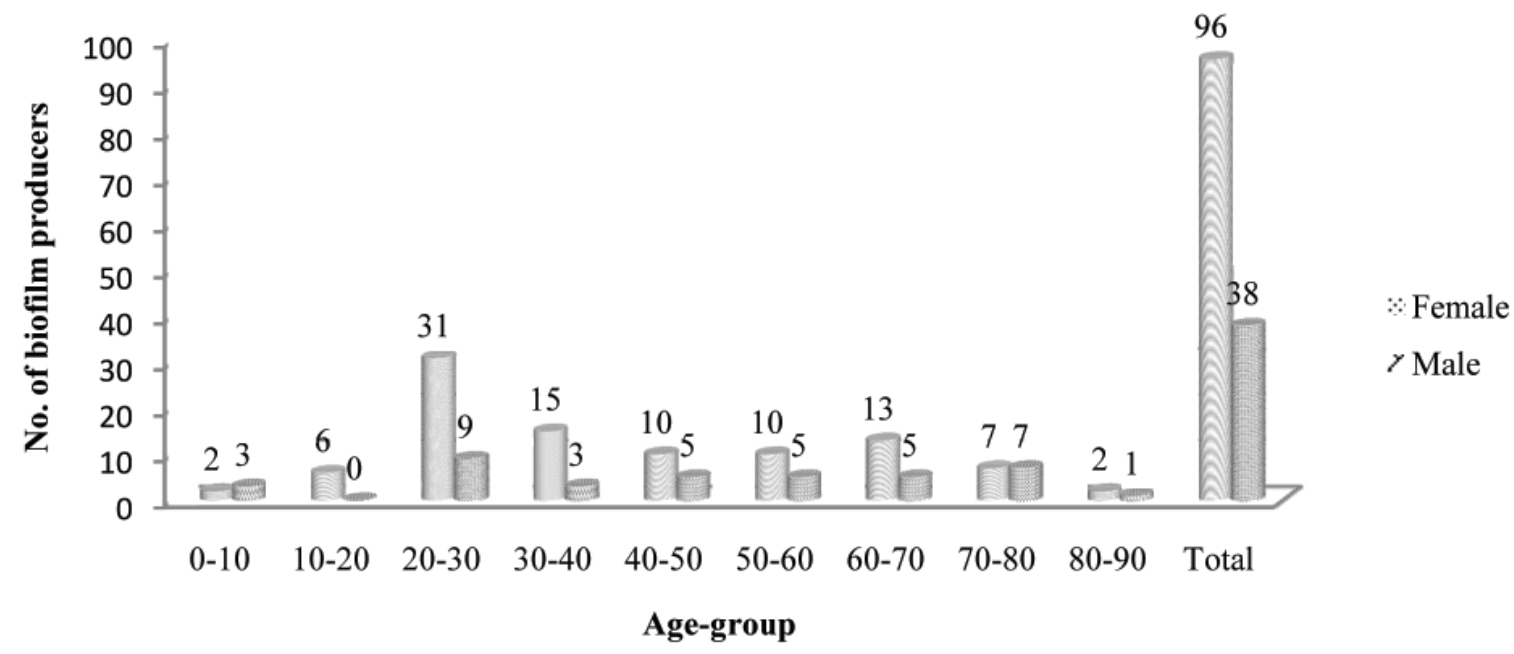

Figure 2: Occurence of biofilm producers among patients

Although, higher resistance was observed for individual antibiotics tested for biofilm producing and biofilm non- producing uropathogens, difference in antibiotic resistance pattern was not statistically significant for majority of the antibiotics tested. 
Table 3: Antibiotic resistance among biofilm producing and non producing uropathogens

\begin{tabular}{|c|c|c|c|}
\hline \multirow{2}{*}{ Antibiotic } & \multicolumn{3}{|c|}{ Resistance among uropathogens } \\
\hline & Biofilm Producers $(n=134)$ & Biofilm non-producer $(n=100)$ & p-value \\
\hline$A M X$ & $105(78.5 \%)$ & $73(73 \%)$ & $>0.05 ; \mathrm{NS}$ \\
\hline CTX & $57(42.5 \%)$ & $33(33 \%)$ & $>0.05$;NS \\
\hline NIT & $3(2.2 \%)$ & $11(11 \%)$ & $<0.05 ; \mathrm{S}$ \\
\hline CIP & $63(47 \%)$ & $36(36 \%)$ & $>0.05 ; \mathrm{NS}$ \\
\hline COT & $61(47.4 \%)(n=129)$ & $41(42.3 \%)(n=97)$ & $>0.05 ; \mathrm{NS}$ \\
\hline CFM & $56(44.4 \%)(n=126)$ & $27(36.4 \%)(n=74)$ & $>0.05 ; \mathrm{NS}$ \\
\hline LEV & $61(45.5 \%)$ & $32(32 \%)$ & $<0.05 ; \mathrm{S}$ \\
\hline NX & $5(62.5 \%)(n=8)$ & $12(52.2 \%)(n=23)$ & $>0.05 ; \mathrm{NS}$ \\
\hline GEN & $3(37.5 \%)(n=8)$ & $5(21.7 \%)(n=23)$ & $>0.05 ; \mathrm{NS}$ \\
\hline $\mathrm{E}$ & $8(100 \%)(n=8)$ & $14(60.9 \%)(n=23)$ & $>0.05 ; \mathrm{NS}$ \\
\hline
\end{tabular}

Among 234 uropathogens, 37.6\% were multidrugresistant. No statistically significant association was observed between biofilm production and multidrug resistance among uropathogens.

Table 4: Association between biofilm production and multi-drug resistance

\begin{tabular}{lccc}
\hline \multicolumn{2}{c}{ Multi-drug Resistance } & Total \\
\hline Biofilm & Yes & No & 134 \\
\hline Producer & $50(37.31 \%)$ & $84(62.68 \%)$ & 100 \\
Non-producer & $38(38 \%)$ & $62(62 \%)$ & 234 \\
\hline Total & $\mathbf{8 8 ( 3 7 . 6 \% )}$ & $\mathbf{1 4 6 ( 6 2 . 4 \% )}$ & \\
\hline
\end{tabular}

p-value $>0.05$

The MIC values of Levofloxacin for the uropathogens ranged from $0.03-16 \mu \mathrm{g} / \mathrm{ml}$ whereas the MBEC values for the biofilm producing urine isolates ranged from 4 to greater than $1024 \mu \mathrm{g} / \mathrm{ml}$. Dramatic increase in the inhibitory concentration on transition from planktonic to sessile forms was observed.

\section{DISCUSSION}

E.coli being the most common agent, accounted for about $78.6 \%$ cases. Diverse virulence factors such as fimbriae, hemolysin, iron uptake systems, cytotoxins, phase variation, biofilm formation, etc act as the weapons of UPEC against the host and help in the establishment of UTI (Davis and Flood 2011).

Biofilm though clinically relevant, its presence is underestimated due to the need for in-vivo diagnosis (Bordi and de Bentzmann 2011). Among the different screening tests available for in- vitro biofilm formation, Modified Congo Red agar method was employed in this study. The Congo red agar method proposed by Freeman et al. (1989) is a simple, cost- effective phenotypic method of screening of biofilm formation and does not require technical expertise, which makes it appropriate for laboratory use in a developing country like ours.

Using the MCRA (Modified Congo Red Agar) method, $57 \%$ of total urinary isolates were found to be biofilm producers. It has been reported that more than $50 \%$ of total human infections are associated with biofilm production (Costerton et al. 1987). Previous studies have also proposed the importance of bacterial biofilm formation in UTI (Chung and Whitefield 2000; Hall et al. 2014). Biofilm is one of the virulence factors of uropathogens allowing them to persist in the urinary tract (Hancock et al. 2007; Marhora et al. 2010). Biofilm provides survival advantage to pathogens through the expression of several other virulence factors, acquisition of antibiotic tolerance and an increased resistance against host immune defenses (Soto et al. 2007).

The incidence of biofilm producers among indoor patients $(62.5 \%)$ was greater than outdoor patients $(56.3 \%)$. However, the distribution of biofilm producers among outdoor and indoor patients was not statistically significant ( $p$ value $>0.05$ ). The debilitated health condition or the use of catheters among indoor patients might account for higher percentage of biofilm producers among indoor patients. From the total E.coli 
isolates, $65 \%$ of UPEC were observed to produce biofilm, which is similar to the findings of Sharma et al. (2009) showing $67.5 \%$ of E.coli isolates as biofilm producers. It has been reported that biofilm forming UPEC are often responsible for the problem of recurrence faced in the case of UTI. As per study of Soto et al. (2007), $74 \%$ of UPEC strains causing relapse was biofilm producers. Antimicrobial Resistance among biofilm producers appeared to be higher as compared to nonbiofilm producers with reference to individual drugs except for Nitrofurantoin. The increase in antimicrobial resistance among biofilm producers is due to slow growth rate and the presence of the protective covering of exopolysaccharide which alters the penetration of antimicrobial agents through the biofilm and hinders the activity of antimicrobial agents against the bacterial cells (Hung and Henderson 2009; Lopez et al. 2010; Hall et al. 2014). The association between biofilm production and multidrug resistance among uropathogens was statistically non-significant. Similar result was obtained by Bardoloi et al. (2014). However, there are a number of literatures available which establish a significant role of biofilm production in multidrug-resistance (Ghanwante 2012; Sanchez et al. 2013).

In this study, MIC was determined for all the uropathogenic isolates and MBEC was determined for all the biofilm forming uropathogens. MBEC provides a more reliable method for accessing the antibiotic susceptibility of biofilm as it is targeted against biofilm mode of life (Ghanwante, 2012). The antibiotic chosen for the determination of MIC and MBEC was Levofloxacin. Levofloxacin is a broad spectrum antibiotic of Fluoroquinolone group. Fluoroquinolones are initial agents in empirical therapy for various types of UTI (McGregor et al. 2008). Moreover, fluoroquinolone are reported to have high clinical cure rates (Akram et al. 2007) as well as have activity against biofilms (Ishida et al. 1998). The MIC values ranged from less than 0.003$16 \mu \mathrm{g} / \mathrm{ml}$. However, the MBEC values were found to be higher and ranged from 2-more than $1024 \mu \mathrm{g} / \mathrm{ml}$. This showed that very high concentration of antibiotic is required for the elimination of biofilm. Studies have revealed that concentration required to inhibit biofilm is 10-1000 times greater than required to inhibit planktonic cells (Chung and Whitefield 2000; Kostakioti et al. 2013). As long as the biofilm associated with any kind of infection is not removed, there is chance that the infection will persist or recur even after the eradication of planktonic cells after the standard antibiotic therapy as the surviving biofilm act as reservoir of pathogenic organisms (Lewis 2001). Since, very high concentration of antibiotic is required for the eradication of biofilms, which is not possible to be obtained in the human body, it is necessary to search for other therapeutic interventions for biofilm associated infections.

\section{CONCLUSION}

The distribution of biofilm forming uropathogens was independent of sex, age and indoor-outdoor distribution of patients. High prevalence of biofilm producers among uropathogens is indicative of the need for screening of biofilm as a common laboratory procedure. Increased antibiotic resistance was observed among biofilm producers as compared to non-biofilm producer strains. The drastic increase in the MBEC as compared to MIC demonstrated high antimicrobial resistance among biofilm producers.

\section{ACKNOWLEDGEMENTS}

We are grateful to Department of Pathology of Kathmandu Model Hospital and Department of Microbiology of GoldenGate International College for providing the necessary support for the completion of this research work.

\section{CONFLICT OF INTEREST}

The authors declare no conflict of interest.

\section{REFERENCES}

Akram M, Shahid M and Khan AU (2007). Etiology and antibiotic resistance patterns of communityacquired urinary tract infections in JNMC Hospital Aligarh, India. Ann Clin Microbial Antimicrob 6:1-7

Bordi C and de Bentzmann S (2011). Hacking into bacterial biofilms: a new therapeutic challenge. Ann Intensive Care 1: 1-8.

Choong S and Whitfield H (2000). Biofilms and their role in infections in urology. BJU international 86: 935-941.

CLSI (2013). Performance standards for antimicrobial susceptibility testing; twenty-second informational supplement, vol. 33. CLSI Document M100-S20. Wayne, PA.

Costerton JW, Cheng KJ, Geesey GG, Ladd TI, Nickel JC, Dasgupta M and Marrie TJ (1987). Bacterial biofilms in nature and disease. Ann Rev Microbiol 41: 435-464. 
Donlan RM and Costerton JW (2002). Biofilms: survival mechanisms of clinically relevant microorganisms. Clin Microbiol Rev 15: 167-193.

Ejrnaes K, Stegger M, Reisner A, Ferry S, Monsen T, Holm SE and Frimodt-Møller N (2011). Characteristics of Escherichia coli causing persistence or relapse of urinary tract infections: phylogenetic groups, virulence factors and biofilm formation. Virulence 2:528-537.

EUCAST (2000). Determination of minimum inhibitory concentrations (MICs) of antibacterial agents by agar dilution: EUCAST definitive document 3.1. Clin Microbiol Infect 6: 509-516.

Freeman DJ, Falkiner FR and Keane CT (1989). New method for detecting slime production by coagulase negative staphylococci. J Clinical Path 42: 872-874.

Ghanwate NA (2012). Biofilm eradication studies on uropathogenic E. coli using ciprofloxacin and nitrofurantoin. Int J Pharm Biomed Res 3: 127-131.

Hall MR, McGillicuddy E and Kaplan LJ (2014). Biofilm: basic principles, pathophysiology, and implications for clinicians. Surg Infect 15 :1-7.

Hancock V, Ferrieres L and Klemm P (2007). Biofilm formation by asymptomatic and virulent urinary tract infectious Escherichia coli strains. FEMS Microbiol Lett 267: 30-37.
Hung CS and Henderson JP (2008). Emerging concepts of biofilms in infectious diseases. Missouri Medicine. 106: 292-296.

Ishida H, Ishida Y, Kurosaka Y, Otani T, Sato K and Kobayashi $H$ (1998). In vitro and in vivo activities of levofloxacin against biofilm-producing Pseudomonas aeruginosa. Antimicrob Agents Chemother 42: 1641-1645.

Kostakioti M, Hadjifrangiskou M and Hultgren SJ (2013). Bacterial biofilms: development, dispersal, and therapeutic strategies in the dawn of the postantibiotic era. Cold Spring Harb Perspect Med 3: 1-23.

Lewis K (2001). Riddle of biofilm resistance. Antimicrob Agents Chemother 45: 999-1007.

McGregor JC, Allen GP and Bearden DT (2008) Levofloxacin in the treatment of complicated urinary tract infections and acute pyelonephritis. Ther Clin Risk Manag 4: 843-853.

Sharma M, Aparna, Yadav S, Chaudhary U (2009). Biofilm production in uropathogenic Escherichia coli. Indian J Pathol Microbiol. 52: 294-298.

Soto SM, Smithson A, Martinez JA, Horcajada JP, Mensa J and Vila J (2007). Biofilm formation in uropathogenic Escherichia coli strains: relationship with prostatitis, urovirulence factors and antimicrobial resistance. J Urol 177: 1387-1392. 